\title{
SERIE DE CASOS \\ Manejo de masas anexiales benignas por laparoscopia: Experiencia en la unidad de endoscopia ginecológica del Hospital Infantil Universitario Lorencita Villegas de Santos (1995-1999)
}

\author{
Camilo Rueda Beltz*, Felipe Arbeláez Casas MD**, Ángela María Ruiz Sternberg MD***
}

Recibido: Nov. 15/2000 - Revisado: Enero 29/2001 - Aceptado: Mayo 11/2001

\begin{abstract}
RESUMEN
Introducción: La información que en la actualidad muestra la literatura mundial, acerca del manejo de las masas anexiales benignas por vía laparoscópica, al compararla con la laparotomía, resalta las bondades de la laparoscopia en importantes aspectos como son el costo, la estancia hospitalaria y el tiempo de convalecencia.

Metodología: Se realizó un estudio descriptivo, bidireccional, serie de casos con 120 pacientes que fueron llevadas a laparoscopia operatoria, por sospecha diagnóstica de masa anexial benigna, en la Unidad de Endoscopia Ginecológica del Hospital Infantil Universitario Lorencita Villegas de Santos, durante el período comprendido entre noviembre de 1995 y marzo de 1999. Se analizaron tanto variables clínicas como paraclínicas y se correlacionaron con el resultado de histopatología. Los datos fueron procesados mediante Excel versión 7.0, obteniendo los porcentajes y la desviación estándar en cada una de las variables numéricas analizadas.

Resultados: El principal motivo de consulta fue el dolor pélvico crónico presentado en 68,33\%. Dentro del grupo de pacientes estudiadas no se presentó ningún caso de malignidad y el tipo histológico más frecuente correspondió al quiste de paraovario en 30,8\%. El rango de tamaño de las masas por ultrasonografía estuvo entre $4,73 \mathrm{~cm}+/-1,37$ y $5,47+/-1,29$. El resultado del Ca 125 fue anormal en el $40 \%$ de los endometriomas, en el $12,5 \%$ de los teratomas quísticos maduros y en el $22,2 \%$ del grupo clasificado como "otros". No se registraron complicaciones intraoperatorias ni postoperatorias.

Discusión: En este estudio, la adecuada evaluación prequirúrgica e intraoperatoria de las masas anexiales, permitió en todos los casos llevar estas a cirugía con una alta presunción de benignidad, resultado que se corroboró en la patología definitiva. La laparoscopia es una vía segura para abordar las masas anexiales sugestivas de benignidad y se puede considerar como una alternativa respecto a la laparotomía.
\end{abstract}

Palabras clave: laparoscopia, masas anexiales.

\footnotetext{
* Residente III de Ginecología y Obstetricia. Universidad del Rosario _ Hospital Infantil Universitario Lorencita Villegas de Santos ** Jefe Unidad de Endoscopia Ginecológica Hospital Infantil Universitario Lorencita Villegas de Santos)

*** Ginecóloga Endoscopista Unidad de Endoscopia Ginecológica Hospital Infantil Universitario Lorencita Villegas de Santos
}

Art. 010 


\begin{abstract}
Introduction: The information currently shown in literature worldwide regarding the handling of benign annexed masses way laparoscopy, as opposed to laparotomy, enhances the benefits of laparoscopy in important issues such as cost, hospitalization and convalescence period.

Methodology: A descriptive, bi-directional study involving a series of cases was entered undertaken with 120 patients that were taken to laparoscopy surgery due to suspicious diagnosis of benign annexed mass at the Unit of Gynecological Endoscopy of Hospital Infantil Universitario Lorencita Villegas de Santos (Children's Hospital of Bogotá). Such study was done between November 1995 and March 1999. Both clinical and paraclinical variables were analyzed and correlated to histopathological results. Data was processed on Excel version 7.0, having obtained the percentages and standard deviations of each of the analyzed numerical variables.
\end{abstract}

\title{
Results
}

The main cause of consultation was the chronic pelvic pain shown by a $68.33 \%$. With the group of patients studied there were no malignant cases and the most frequent histological type corresponded to parovarian cyst in $30.8 \%$. The size range of ultrasonic masses was between $4.73 \mathrm{~cm}+/-1.37$ and 5.47 +/- 1.29. Results of Ca 125 were abnormal within $40 \%$ of endometriomas, $12.5 \%$ of mature cystic teratomas and $22.2 \%$ of the group classified as "others". There were no intra-surgery or post-surgery complications.

\section{Discussion}

This study's adequate pre-surgical and intra-surgical evaluation of annexed masses allowed all cases to be practiced surgery with a high presumption of benignity, results corroborated by the final pathology. Laparoscopy is a safe way to address benign-suggestive annexed masses.

Key words: laparoscopy, annexed masses.

\section{INTRODUCCIÓN}

El acceso quirúrgico tradicional para extirpar un tumor pélvico ha sido la laparotomía, con el propósito de resecar y evaluar adecuadamente el estadío de cáncer ovárico, cuando se encuentra. Sin embargo casi todos los tumores anexiales son benignos y se encuentran lesiones malignas sólo en el 7 a $13 \%$ de las pacientes premenopáusicas y 8 a $45 \%$ de las postmenopáusicas. Una encuesta realizada en 1988 por la Asociación Americana de Ginecólogos Laparoscopistas (AAGL) evidenció que de 36928 laparoscopias operatorias, 5075 habían sido por lesiones ováricas, constituyéndose en esa época en la tercera causa de este procedimiento. Dos años después, esta misma asociación encontró triplicada esta indicación (13.739), donde el $96 \%$ de estas pacientes fueron premenopáusicas ${ }^{1}$.

Los principales aspectos a tomar en cuenta al considerar la laparoscopia quirúrgica como una alternativa razonable en la valoración de pacientes con lesiones ováricas son, el estudio cuidadoso preoperatorio y los pasos obligatorios durante el transoperatorio con el fin de detectar patología maligna.
Con este estudio, se pretende mostrar la experiencia de la Unidad de Endoscopia Ginecológica (UEGO) del Hospital Infantil Universitario Lorencita Villegas de Santos (HIULVS) en 120 pacientes con masas anexiales sugestivas de benignidad, durante el período comprendido entre noviembre de 1995 y marzo de 1999.

\section{MATERIALES Y MÉTODOS}

Criterios de inclusión: Durante el período comprendido entre noviembre de 1995 y marzo de 1999, se incluyeron las historias clínicas de las pacientes que consultaron a la Unidad de Endoscopia Ginecológica (UEGO) del Hospital Infantil Universitario Lorencita Villegas de Santos, con diagnóstico de masa anexial. Todas debían presentar hallazgos clínicos y paraclínicos de presunción de benignidad, como: ausencia de cáncer de ovario previo, criterios ecográficos que mostraran masa anexial menor de $12 \mathrm{~cm}$, pared menor de $3 \mathrm{~mm}$, ausencia de papilas, de septos, baja ecogenicidad y marcadores tumorales negativos; estos últimos solicitados de acuerdo a la condición clínica de cada paciente.

Se excluyeron las pacientes que presentaron contraindicación para la realización de la laparoscopia, 
como enfermedad pulmonar severa, enfermedad gastrointestinal obstructiva, hernia diafragmática y las irradiaciones pélvicas o abdominales previas.

Todos los procedimientos, fueron practicados en la Unidad de Endoscopia Ginecológica (UEGO) del Hospital Infantil Universitario Lorencita Villegas de Santos, por los ginecólogos laparoscopistas de la institución. Los estudios de anatomía patológica, las biopsias por congelación y la evaluación definitiva se realizaron siempre por los mismos patólogos del hospital.

Todas las pacientes seleccionadas para el estudio, fueron debidamente informadas del procedimiento y se contó con su previa autorización escrita. Se les informó sobre las posibilidades quirúrgicas y los riesgos de concluir el procedimiento con una cirugía radical, en el caso en que la biopsia por congelación reportara una lesión limítrofe o maligna y de acuerdo con la condición clínica de la paciente.

A todas las pacientes se les realizó una detallada historia clínica, se les solicitó una ecografía pélvica, que debía cumplir con los criterios sonográficos de presunción de benignidad descritos por Sassone ${ }^{13}$. Igualmente, se solicitaron marcadores tumorales como el Ca125, alfa fetoproteína, beta hCG y antígeno carcinoembrionario, de acuerdo a cada caso y con el fin de determinar el comportamiento biológico de la lesión anexial.

En el acto quirúrgico, la laparoscopia se realizó según los protocolos del departamento, con una punción principal umbilical por técnica abierta de $10 \mathrm{~mm}$ y 2 o 3 puertos accesorios de 5 o $12 \mathrm{~mm}$ en fosas ilíacas y suprapúbicas según fuese necesario. Durante el procedimiento, se realizó una evaluación detallada de la cavidad abdomino-pélvica, toma de citologías de líquido peritoneal y del tumor cuando su apariencia fue quística, análisis del anexo contralateral y de la lesión propiamente dicha, estudio tanto macroscópico (por medio de cistoscopia o eversión del quiste para verificar presencia de papilas, tabiques o engrosamientos de la pared) como microscópico, biopsia por congelación.

La decisión quirúrgica dependió del resultado de la biopsia por congelación y de las características clínicas de cada paciente. El seguimiento postoperatorio, se realizó a las dos semanas para descartar complicaciones y para evaluar el resultado definitivo de la anatomía patológica. Posteriormente, se citó a las pacientes, una vez por año a partir de la cirugía, al servicio de consulta externa de la UEGO y se interrogó acerca de la persistencia o desaparición de la sintomatología previa al procedimiento; se realizó nuevamente un examen físico ginecológico y se solicitaron ecografía pélvica y marcadores tumorales, de acuerdo a la condición clínica de cada paciente para evaluar la reincidencia del tumor de ovario. Igualmente se evaluó la incidencia de fertilidad.

Este seguimiento clínico finalizó en marzo de 1999 a raíz del cierre del hospital.

\section{RESULTADOS}

Se incluyeron 120 pacientes desde noviembre de 1995 hasta marzo de 1999. Los datos fueron procesados mediante Excel versión 7.0, obteniendo la desviación estándar en cada una de las variables numéricas analizadas.

En cuanto a las características demográficas de la población, se encontró un rango de edad entre 17-59 años con un promedio de $32,6+/-8,65$, donde el promedio de menor edad correspondió al grupo de pacientes con quistes funcionales $28,5+/-7,21$ y las

Tabla 1.

Correlación entre características demográficas y resultado de histopatología

\begin{tabular}{|c|c|c|c|c|c|c|c|}
\hline \multirow[b]{2}{*}{ TIPO HISTOLÓGICO } & \multicolumn{3}{|c|}{ EDAD } & \multirow{2}{*}{\multicolumn{2}{|c|}{$\begin{array}{l}\text { NULIPARIDAD } \\
(\mathrm{N})\end{array}$}} & \multirow{2}{*}{\multicolumn{2}{|c|}{ MULTIPARIDAD (M) }} \\
\hline & RANGO & PROMEDIO & $\begin{array}{c}\text { DESV. } \\
\text { ESTANDAR }\end{array}$ & & & & \\
\hline Quiste paraovario & $18-55$ & 32 & 9.19 & 15 & $40.54 \%$ & 22 & $59.46 \%$ \\
\hline Endometrioma & $23-43$ & 33.4 & 5.81 & 13 & $46.43 \%$ & 15 & $53.57 \%$ \\
\hline Quiste simple & $17-55$ & 33.27 & 12.4 & 2 & $18.18 \%$ & 9 & $81.82 \%$ \\
\hline Quiste funcional & $18-41$ & 28.5 & 7.21 & 9 & $75 \%$ & 3 & $25 \%$ \\
\hline Teratoma quístico maduro & $20-40$ & 31.7 & 5.67 & 1 & $7.69 \%$ & 12 & $92.31 \%$ \\
\hline Cistadenoma seroso & $19-38$ & 29.8 & 7.69 & 4 & $80 \%$ & 1 & $20 \%$ \\
\hline Otros & $20-59$ & 36.2 & 11.45 & 6 & $42.86 \%$ & 8 & $57.14 \%$ \\
\hline Total & $17-59$ & 32.6 & 8.65 & 50 & $41.67 \%$ & 70 & $58.33 \%$ \\
\hline
\end{tabular}




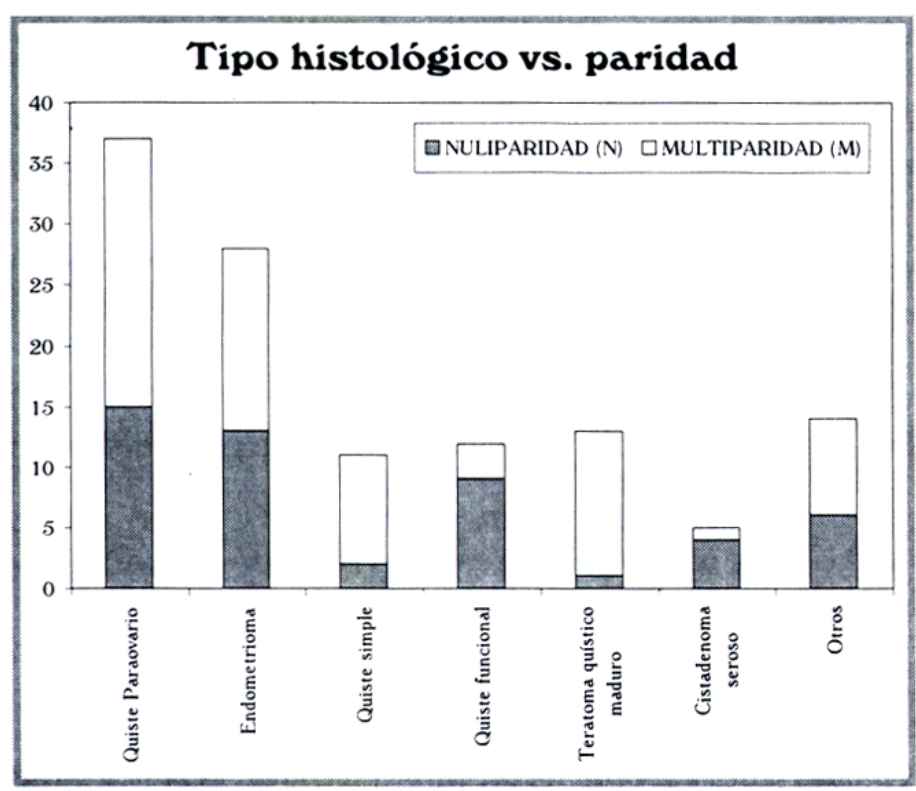

Fig 010i01

Tabla 2.

Frecuencia motivos de consulta

\begin{tabular}{|lcr|}
\hline \multicolumn{1}{|c|}{ MOTIVO DE CONSULTA } & $\mathrm{n}$ & \multicolumn{1}{c|}{$\%$} \\
\hline D.P.C. & 82 & $68.33 \%$ \\
H.U.A. & 9 & $7.50 \%$ \\
Infertilidad & 6 & $5 \%$ \\
Asintomáticas & 23 & $19.17 \%$ \\
\hline Total & 120 & $100 \%$ \\
\hline
\end{tabular}

pacientes de mayor edad promedio se encontraron en el grupo clasificado como "otros", donde esta fue de 36,2 +/- 11,45. De las 120 pacientes del estudio, 50 fueron nulíparas $(41,67 \%)$ y 70 fueron multíparas $(58,33 \%)$. Tabla I.

El principal motivo de consulta fue el dolor pélvico crónico presentado por 82 pacientes $(68,33 \%), 23$ fueron asintomáticas correspondiendo al 19,17\%. Tabla II.

Dentro del grupo de pacientes estudiadas no se presentó ningún caso de malignidad en la histopatología. Un caso correspondiente a cistoadenoma mucinoso, resultó ser border line, en el informe definitivo de patología, razón por la cual, se decidió reintervenir a la paciente y completar la cirugía. El tipo histológico más frecuente correspondió al quiste de paraovario, con 37 casos $(30,8 \%)$ y el menos encontrado fue el cistoadenoma seroso, visto en 5 pacientes $(4,17 \%)$ Tabla III.

Se tuvieron en cuenta parámetros tanto clínicos como paraclínicos en la correlación de la evolución prequirúrgica y el resultado de histopatología. El exámen

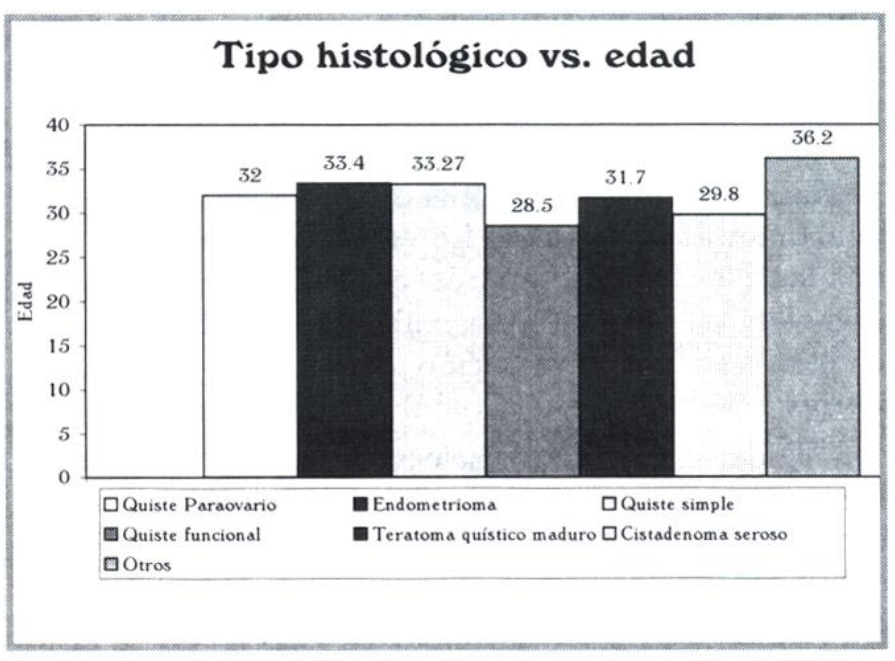

Fig 010i02

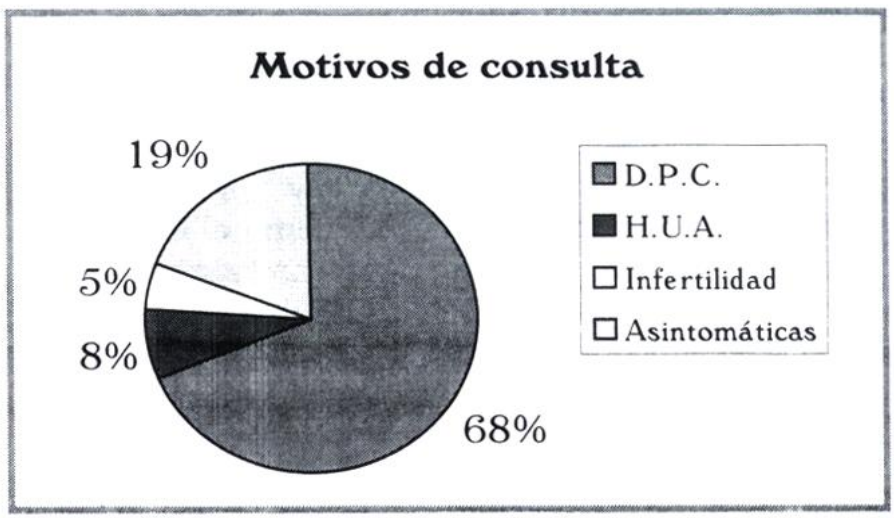

Fig $010 \mathrm{i} 03$

físico se clasificó como anormal cuando se encontró la masa en la valoración ginecológica; por tacto vaginal y normal, cuando no se encontró masa anexial. Tabla IV.

El rango del tamaño de las masas por ultrasonografía estuvo entre $4,73 \mathrm{~cm}+/-1,37$ y $5,47 \mathrm{~cm}+/-1,29$. El hallazgo predominante en la ecogenicidad fue el de componente mixto, seguido del quístico y posteriormente el sólido. Dentro de los diferentes tipos histológicos, el mayor porcentaje de masas quísticas se encontró en el grupo del quiste de paraovario $83,87 \%$, los endometriomas fueron los que tuvieron en mayor porcentaje una apariencia mixta $(53,85 \%)$ y el teratoma quístico maduro fue el tumor que mas se encontró como sólido en la valoración ecográfica. (40\%) Tabla V.

Se realizaron marcadores tumorales a 89 pacientes. $(74,17 \%)$ Tanto la AFP, como la BHCG y el ACE, resultaron normales en todos los casos en los que se practicaron. Al contrario de los anteriores, el comportamiento del $\mathrm{Ca} 125$ fue diferente. Fue anormal (>35U/ $\mathrm{ml})$ en 9 endometriomas $(40,91 \%)$, en un teratoma 
Tabla 3.

Frecuencia de tipos histológicos

\begin{tabular}{|lcr|}
\hline \multicolumn{1}{|c}{ TIPO HISTOLÓGICO } & $\mathrm{n}$ & $\%$ \\
\hline Quiste Paraovario & 37 & $30.83 \%$ \\
Endometrioma & 28 & $23.33 \%$ \\
Quiste simple & 11 & $9.17 \%$ \\
Quiste funcional & 12 & $10 \%$ \\
Teratoma quístico maduro & 13 & $10.83 \%$ \\
Cistadenoma seroso & 5 & $4.17 \%$ \\
Otros & 14 & $11.67 \%$ \\
\hline Total & 120 & $100 \%$ \\
\hline
\end{tabular}

Tabla 4.

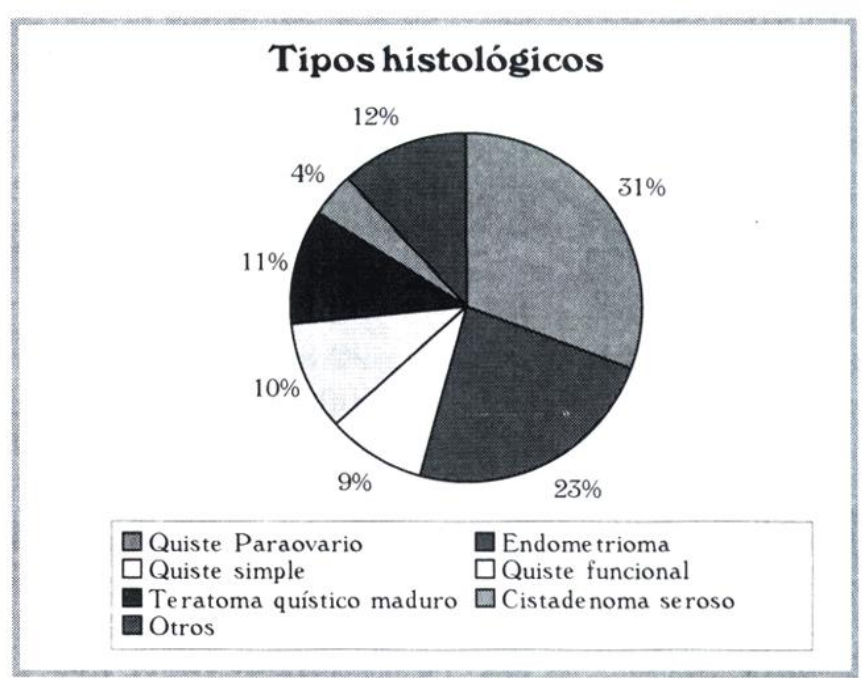

Fig $010 \mathrm{i} 04$

Evaluación preoperatoria y resultado de histopatología

\begin{tabular}{|c|c|c|c|c|c|c|}
\hline \multirow{2}{*}{ TIPO HISTOLÓGICO } & \multicolumn{6}{|c|}{ EXAMEN FISICO } \\
\hline & \multicolumn{2}{|c|}{ NORMAL } & \multicolumn{2}{|c|}{ ANORMAL } & \multicolumn{2}{|c|}{ TOTAL } \\
\hline Quiste Paraovario & 13 & $35.14 \%$ & 24 & $64.86 \%$ & 37 & $100 \%$ \\
\hline Endometrioma & 10 & $35.71 \%$ & 18 & $64.29 \%$ & 28 & $100 \%$ \\
\hline Quiste simple & 2 & $18.18 \%$ & 9 & $81.82 \%$ & 11 & $100 \%$ \\
\hline Quiste funcional & 6 & $50 \%$ & 6 & $50 \%$ & 12 & $100 \%$ \\
\hline Teratoma quístico maduro & 5 & $38.46 \%$ & 8 & $61.54 \%$ & 13 & $100 \%$ \\
\hline Cistadenoma seroso & 1 & $20 \%$ & 4 & $80 \%$ & 5 & $100 \%$ \\
\hline Otros & 5 & $35.71 \%$ & 9 & $64.29 \%$ & 14 & $100 \%$ \\
\hline
\end{tabular}

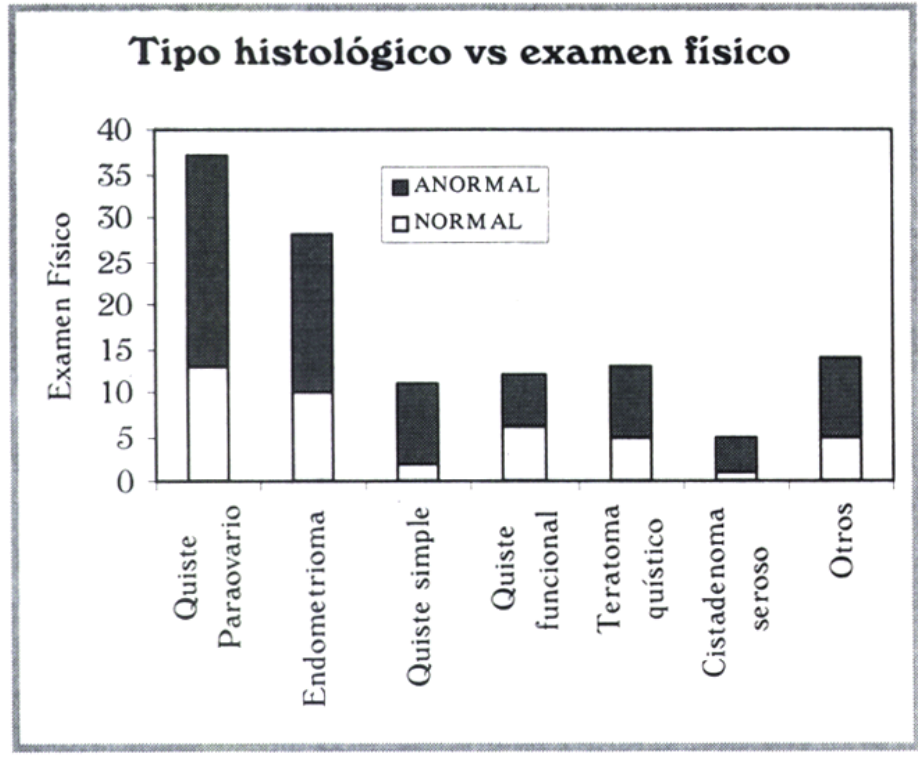

Fig $010 \mathrm{i} 05$ quístico maduro $(12,5 \%)$ y en 2 casos clasificados como "otros", (1 cistadenoma mucinoso y 1 quiste del cuerpo lúteo) correspondiendo a un 22,22\%. Tabla VI.

El promedio de costos por paciente fue de $\$ 482,109.28+$ +/ $\$ 303,459.22$ y los días de estancia hospitalaria en promedio fueron de $1,8+/-0,96$. No se registraron complicaciones $\mathrm{ni}$ en el procedimiento quirúrgico, ni en el post-operatorio de ninguna paciente. Tabla VII.

En el estudio 112 pacientes pertenecieron al grupo de edad fértil (posterior a la menarquia y antes de la menopausia) y 8 pacientes fueron post-menopáusicas. La cirugía conservadora, es decir preservar el ovario, se realizó a 84 pacientes del grupo de edad fértil(75\%).No se realizó cirugía conservadora a ninguna paciente postmenopáusica, donde se realizó salpingo-oforectomía bilateral. La cirugía no conservadora se realizó en 28 pacientes en edad fértil $(25 \%)$ y a la totalidad del grupo 
Tabla 5.

Evaluación preoperatoria y resultado de histopatología

\begin{tabular}{|c|c|c|c|c|c|c|c|c|c|c|}
\hline \multirow{3}{*}{$\begin{array}{c}\text { TIPO } \\
\text { HISTOLÓGICO }\end{array}$} & \multicolumn{10}{|c|}{ ECOGRAFIA } \\
\hline & & & TAMAÑO & DESVIACIÓN & & \multicolumn{5}{|c|}{ ECOGENICIDAD } \\
\hline & \multicolumn{2}{|c|}{ TOTAL } & PROMEDIO & ESTÁNDAR & & Q & & M & & $S$ \\
\hline Quiste Paraovario & $31 / 37$ & $83.78 \%$ & 5.37 & 1.79 & 26 & $83.87 \%$ & 3 & $9.68 \%$ & 2 & $6.45 \%$ \\
\hline Endometrioma & $26 / 28$ & $92.86 \%$ & 4.73 & 1.37 & 10 & $38.46 \%$ & 14 & $53.85 \%$ & 2 & $7.69 \%$ \\
\hline Quiste simple & $11 / 11$ & $100.00 \%$ & 5.24 & 1.45 & 7 & $63.64 \%$ & 4 & $36.36 \%$ & 0 & $0.00 \%$ \\
\hline $\begin{array}{l}\text { Quiste funcional } \\
\text { Teratoma quístico }\end{array}$ & $9 / 12$ & $75.00 \%$ & 5.03 & 1.12 & 5 & $55.56 \%$ & 3 & $33.33 \%$ & 1 & $11.11 \%$ \\
\hline Maduro & $10 / 13$ & $76.92 \%$ & 4.91 & 1.67 & 3 & $30.00 \%$ & 3 & $30.00 \%$ & 4 & $40.00 \%$ \\
\hline $\begin{array}{l}\text { Cistadenoma } \\
\text { seroso }\end{array}$ & $4 / 5$ & $80.00 \%$ & 5.47 & 1.29 & 3 & $75.00 \%$ & 0 & $0.00 \%$ & 1 & $25.00 \%$ \\
\hline Otros & $11 / 14$ & $78.57 \%$ & 5.45 & 1.58 & 7 & $63.64 \%$ & 3 & $27.27 \%$ & 1 & $9.09 \%$ \\
\hline
\end{tabular}

Tipo histológico vs Tamaño promedio

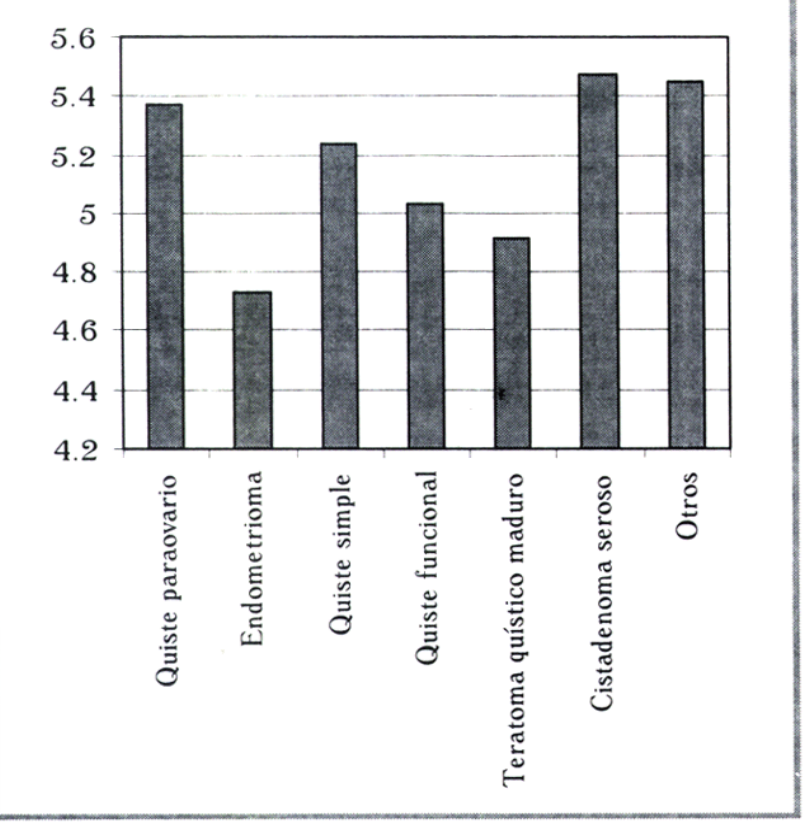

Fig $010 \mathrm{i} 06$

de pacientes postmenopáusicas (8 pacientes 100\%). Tabla VIII.

\section{DISCUSIÓN}

Los tumores anexiales más comúnmente encontrados en mujeres en edad reproductiva son los quistes dermoides o teratomas quísticos maduros, los cuales tienen un porcentaje de bilateralidad entre el 10-15\%. De estos menos del $2 \%$ presentan degeneración maligna, la
Tipo histológico vs ecogenicidad

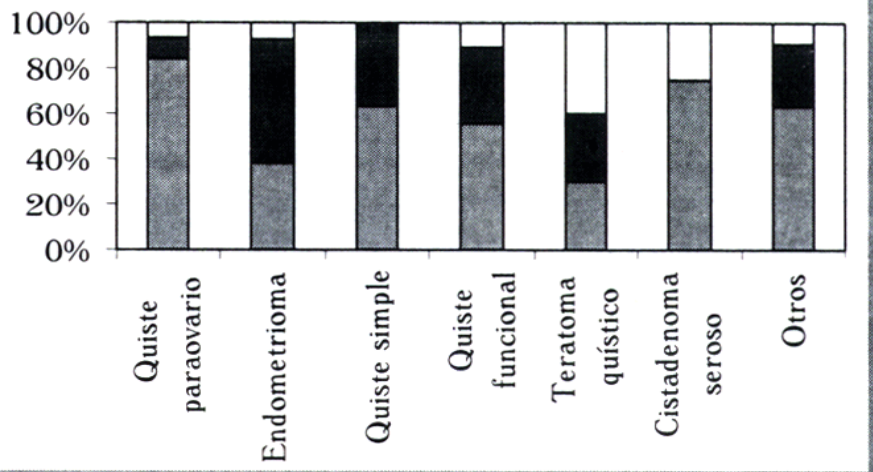

口ECOGENICIDAD Q ECCOGENICIDAD M DECOGENICIDAD S

Fig $010 \mathrm{i} 07$

cual resulta de los elementos escamosos de la masa. Los tumores de las células germinales, son el otro grupo más frecuente de neoplasias en este grupo de edad ${ }^{3}$.

La mayoría de tumores anexiales en pacientes en edad reproductiva son benignos, siendo malignos del 7 al $13 \%$ en pacientes premenopáusicas y del 8 al $45 \%$ en pacientes postmenopáusicas ${ }^{1}$.

En el presente estudio, no se encontró ningúna masa maligna y el más frecuente tipo histológico hallado fue el de quiste de paraovario, en un 30,83\%. El teratoma quístico maduro se encontró en el 10,83\%. La adecuada valoración prequirúrgica e intraoperatoria de las masas, permitió en todos los casos llevar estas a cirugía con una alta presunción de benignidad, resultado que se corroboró en la patología definitiva.

El principal síntoma encontrado en el estudio fue el dolor pélvico crónico en 82 pacientes correspondiendo 


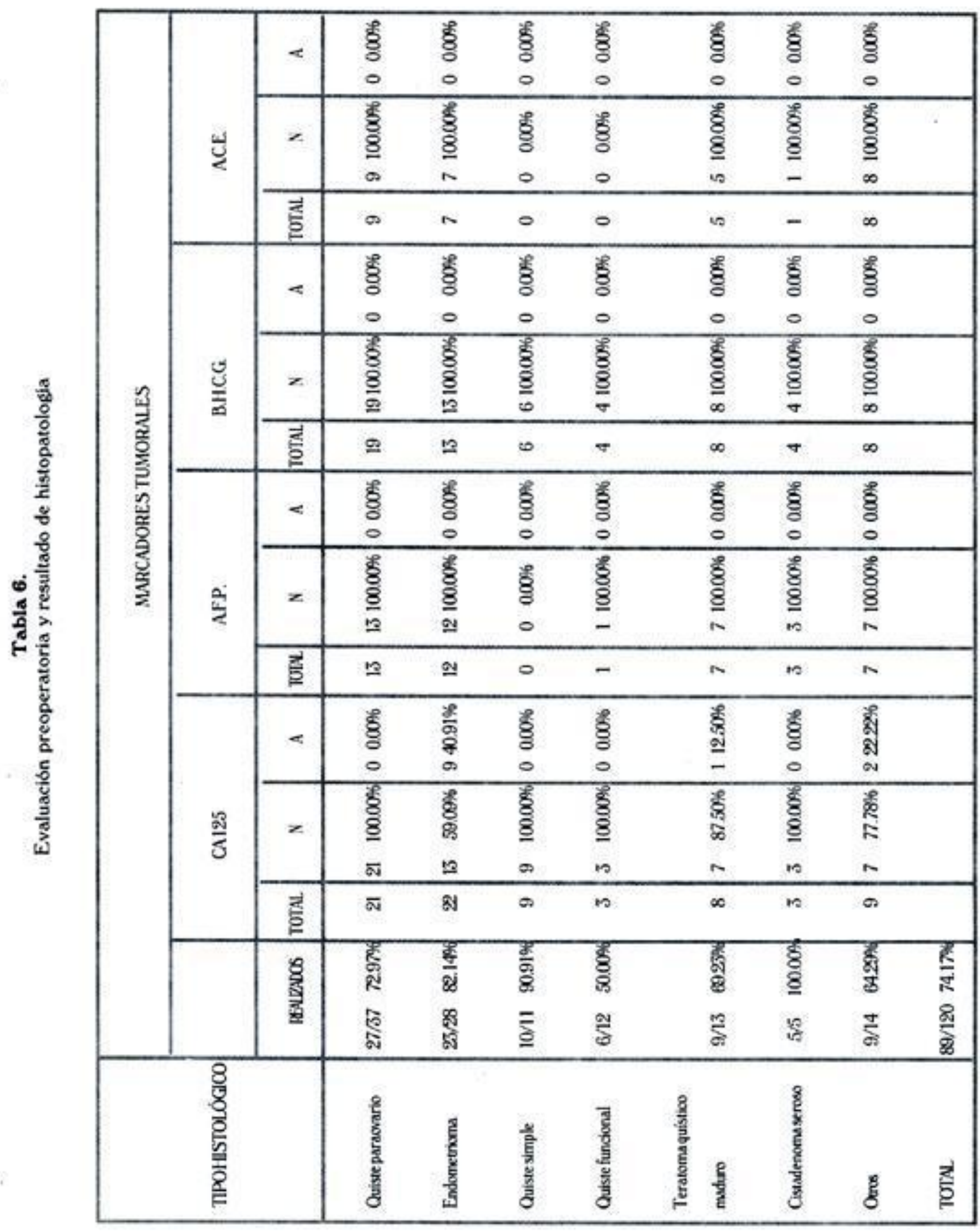




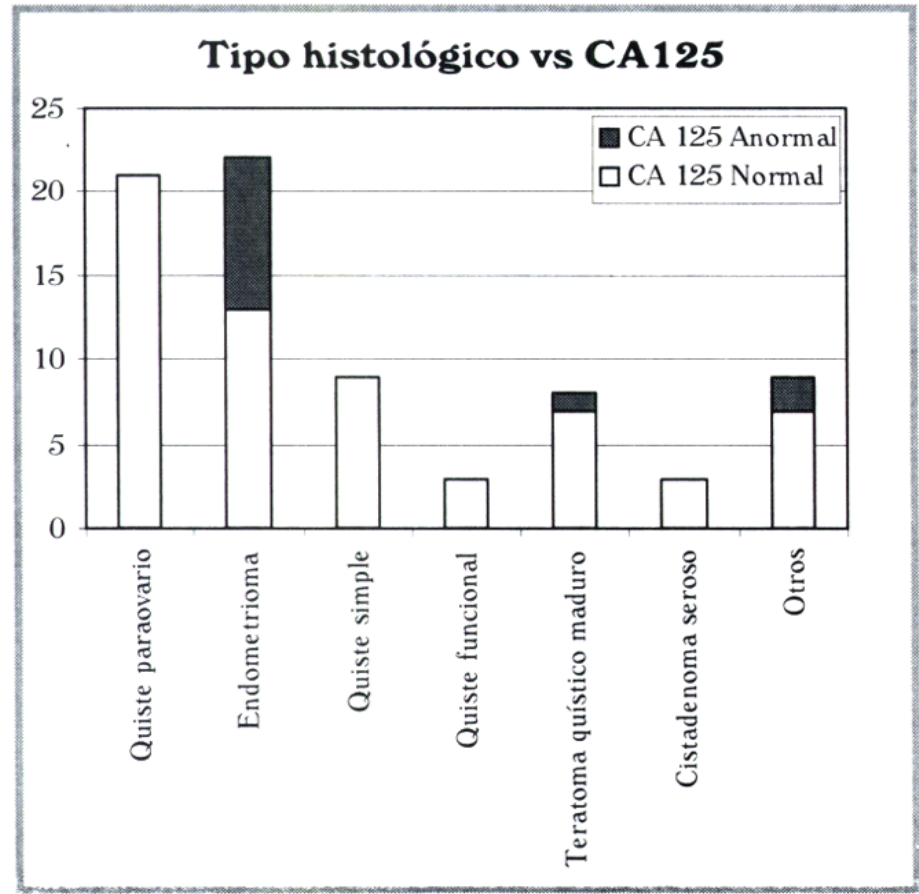

Fig $010 \mathrm{i} 008$

Tipo histológico vs B.H.C.G.

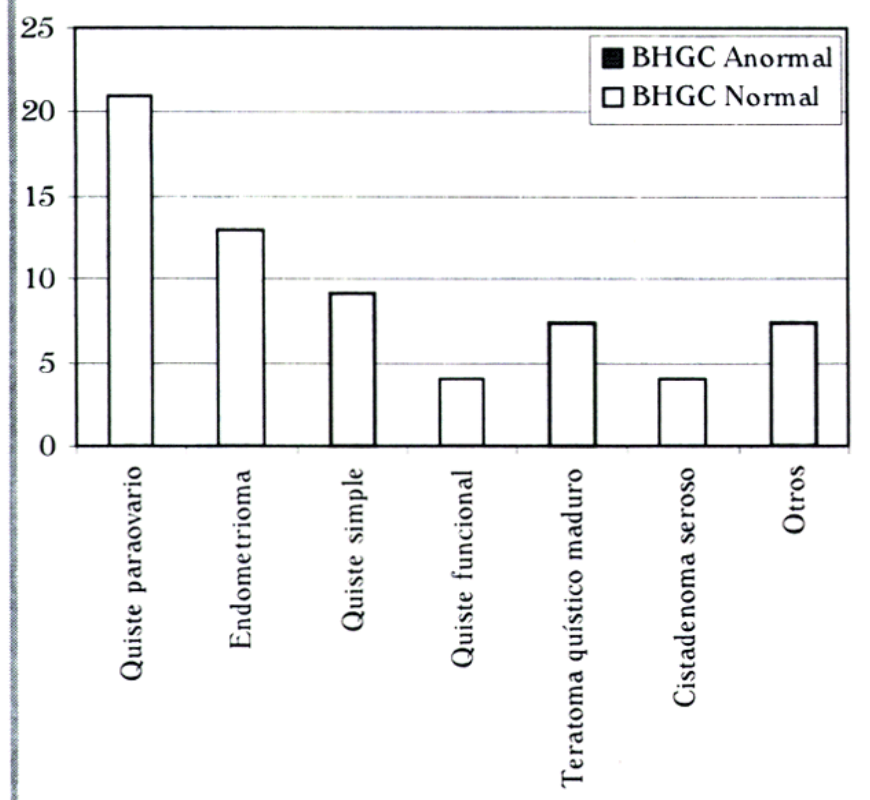

Fig $010 \mathrm{i} 010$

al 68,33\%.El 19,17\% de las pacientes fueron asintomáticas y se llevaron a laparoscopia debido al hallazgo incidental de la masa al examen físico.

Dentro de las herramientas paraclínicas para la valoración preoperatoria de las masas anexiales, la

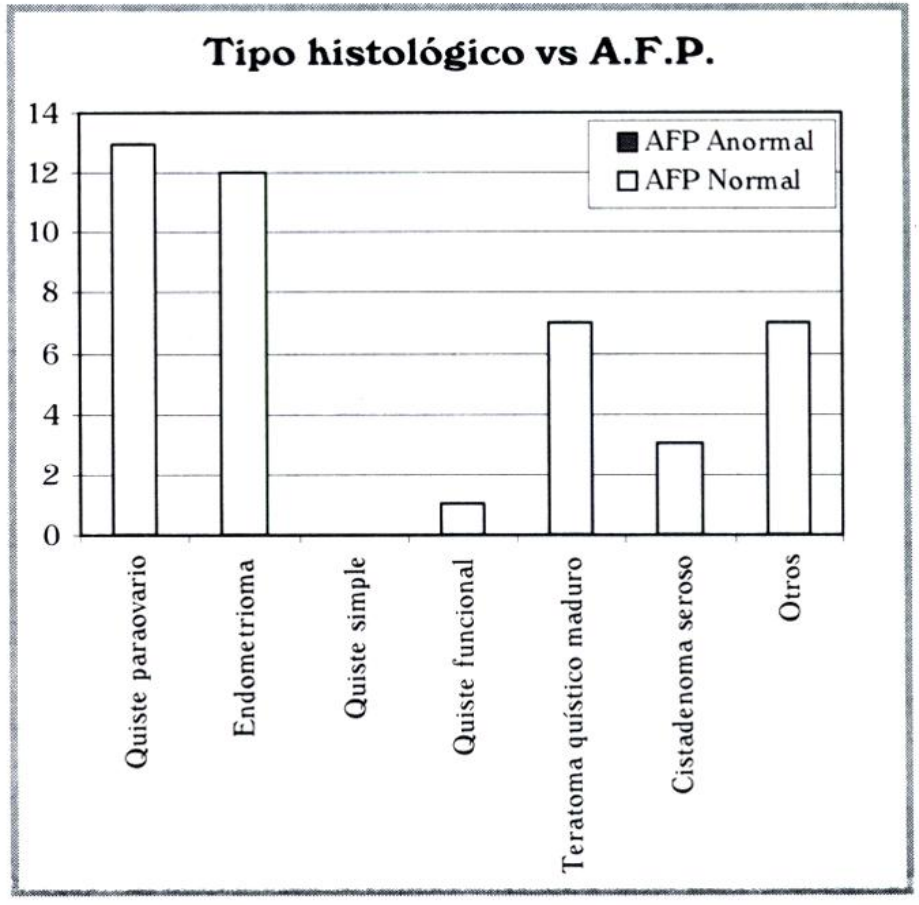

Fig 010i009

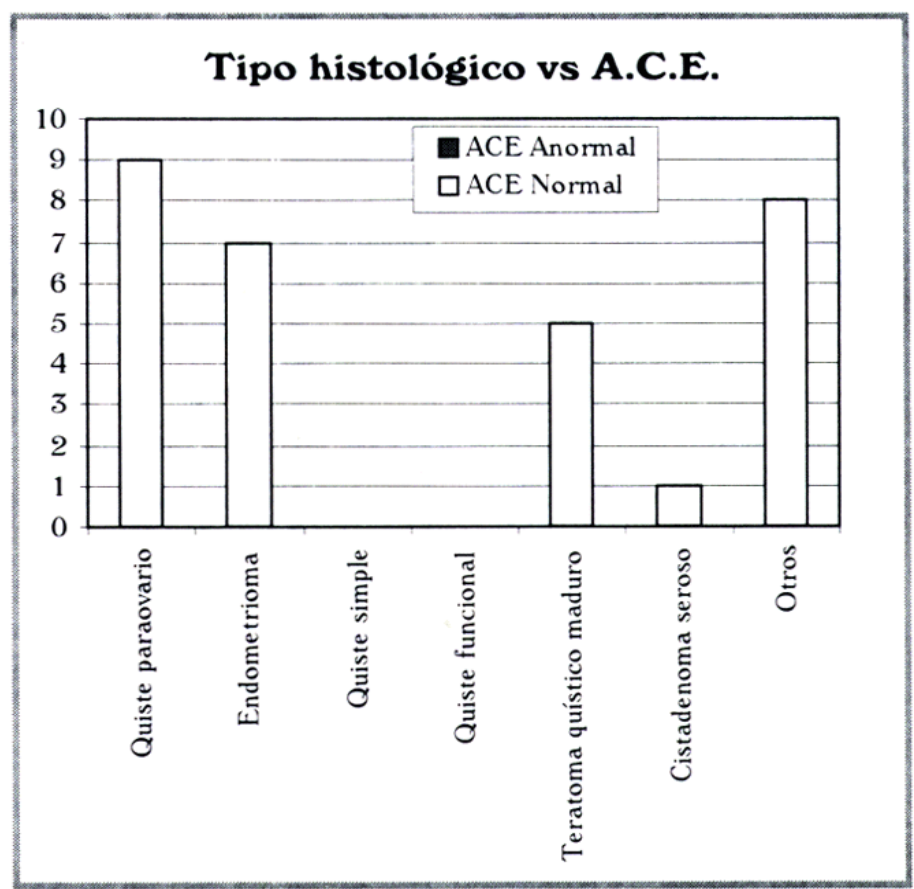

Fig $010 \mathrm{i} 011$

ecogenicidad, la cápsula, la presencia de tabiques y/o papilas y la presencia de líquido libre. Todos estos elementos son de gran utilidad en la aproximación diagnóstica prequirúrgica y tienen un mejor valor 
predictivo cuando se agrupan en puntajes ${ }^{13}$. Las caracvalorar y correlacionar de acuerdo a las características terísticas ecográficas aisladas son importantes para

Tabla 7.

Frecuencia de complicacones, costos y estancia hospitalaria por paciente

\begin{tabular}{lcccccc|}
\hline & $\begin{array}{c}\text { COSTOS } \\
\text { PROMEDIO }\end{array}$ & $\begin{array}{c}\text { DESVIACIÓN } \\
\text { ESTÁNDAR }\end{array}$ & $\begin{array}{c}\text { DÍAS } \\
\text { ESTANCIA }\end{array}$ & $\begin{array}{c}\text { DESVIACIÓN } \\
\text { ESTÁNDAR }\end{array}$ & COMPLICACIONES \\
\hline PARIDAD N & $486,954.77$ & $345,884.88$ & 2,10 & 0,93 & 0 & $0.00 \%$ \\
M & $478,688.93$ & $272,228.08$ & 1,6 & 0,96 & 0 & $0.00 \%$ \\
CX CONSERVADORA & $447,141.88$ & $252,946.92$ & 2 & 0,80 & 0 & $0.00 \%$ \\
CX NO CONSERVADORA & $603,150.00$ & $419,746.73$ & 1,55 & 1,10 & 0 & $0.00 \%$ \\
PREM & 0.00 & & 0 & & 0 & $0.00 \%$ \\
E.F. & $458,736.31$ & $276,875.36$ & 1,83 & 0,86 & 0 & $0.00 \%$ \\
POST & $797,644.38$ & $467,813.68$ & 1,76 & 1,50 & 0 & $0.00 \%$ \\
PROMEDIO PACIENTE & $482,109.28$ & $303,459.22$ & 1,8 & 0,96 & 0 & $0.00 \%$ \\
\hline
\end{tabular}

de cada paciente. Mann y Reich en un estudio de pacientes postmenopáusicas asintomáticas con masa anexial, muestran como la posibilidad de malignidad se incrementa conforme aumenta el tamaño de tumor, siendo de $3 \%$ en masas menores de $5 \mathrm{~cm}$ y hasta de $60 \%$ en masas mayores de $10 \mathrm{~cm}^{12}$.

Tabla 8.

Correlación entre características demográficas y tipo de cirugía

\begin{tabular}{l|cc|cc|}
\hline EDAD & \multicolumn{2}{|c|}{ CX. } & \multicolumn{2}{c|}{ CX. NO } \\
& \multicolumn{2}{c}{ CONSERVADORA } & \multicolumn{2}{c|}{ CONSERVADORA } \\
\hline PREM & 0 & $0 \%$ & 0 & $0 \%$ \\
EF & $84 / 112$ & $75 \%$ & $28 / 112$ & $25 \%$ \\
POST & 0 & $0 \%$ & $8 / 8$ & $100 \%$ \\
\hline TOTAL & $84 / 120$ & & $36 / 120$ & \\
\hline
\end{tabular}

Edad fertil Vs tipo de cirugia

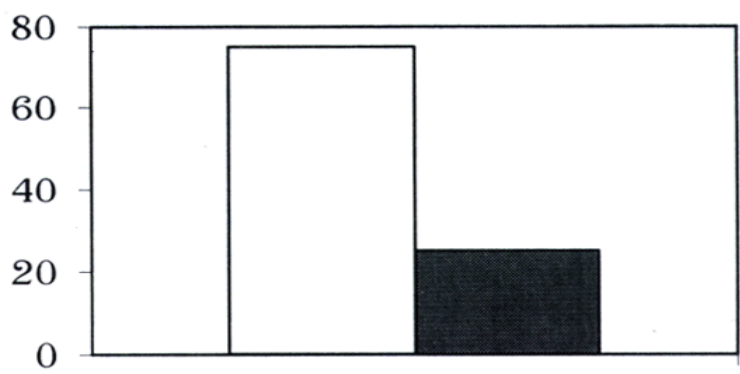

EF

Cirugía conservadora $\square$ Cirugía No conservadora
Los puntajes reunen varios elementos sonográficos. Sassone reporta una sensibilidad para benignidad de $100 \%$, una especificidad de $83 \%$ y un valor predictivo positivo y negativo de $37 \%$ y de $100 \%$ respectivamente ${ }^{13}$ Benacerraf, da una sensibilidad de $80 \%$, especificidad de $87 \%$ y valor predictivo positivo y negativode $73 \%$ y $91 \%{ }^{15}$.

En este estudio, el tamaño ecográfico de las masas estuvo entre $4,73 \mathrm{~cm}+/-1,37$ y $5,47 \mathrm{~cm}+/-1,29$. El componente de la ecogenicidad más común fue el mixto, seguido del quístico y finalmente el sólido. El principal grupo histológico con comportamiento ecográfico hiperecogénico fue el de los teratomas quísticos maduros en un $40 \%$.

Existen diversos tipos de marcadores tumorales utilizados con el fin de aumentar la sensibilidad y especificidad de la valoración preoperatoria de los tumores de ovario. El Ca 125, debido a su origen y comportamiento biológico, se incrementa en diferentes

\section{Postmenopausicas vs tipo de cirugía}

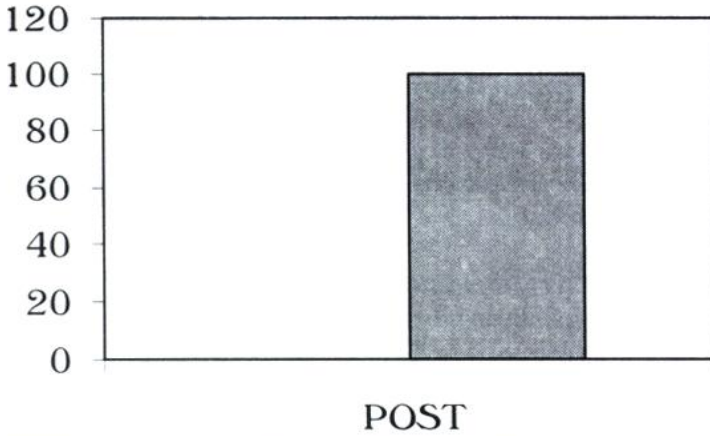

$\square$ Cirugía conservadora $\square$ Cirugía No conservadora 
enfermedades inflamatorias e infecciosas benignas, tanto genitales como extragenitales, así como en masas anexiales benignas. En las pacientes postmenopáusicas con tumor anexial, este marcador serológico gana una mayor importancia, pues cuando este se encuentra elevado existe la probabilidad de $80 \%$ de estar ante un cáncer de ovario $^{8,11}$.

En el presente estudio, se vio elevado en el $40,91 \%$ de los endometriomas, en el $12,5 \%$ de los teratomas quísticos maduros y en el $22 \%$ del grupo clasificado como "otros". Esto se correlaciona con los datos de la literatura donde la principal entidad benigna que cursa con niveles elevados de Ca125 es la endometriosis20. Se observa entonces, la escasa utilidad de este marcador en el estudio de pacientes en edad reproductiva con masas anexiales.

De la mano del estudio prequirúrgico, va la valoración intraoperatoria que es igualmente importante realizar en el manejo de las masas anexiales. Esta incluye la valoración completa de la cavidad abdomino-pélvica, el lavado peritoneal y la citología del mismo, la cistoscopia y citología del líquido del quiste, en el caso en que la masa sea de este aspecto, y finalmente siempre la biopsia por congelación.

A pesar de realizar todos estos pasos de forma rigurosa, existe siempre el riesgo de estar ante una lesión maligna. Nezhat $0,4 \%$, Canis 2,5\%, Asociación Americana de Ginecólogos Laparoscopistas, 0,4\% ${ }^{5-7}$.

Es de resaltar en el presente estudio, la importancia que se le dió a la cirugía conservadora en pacientes en edad fértil (75\%), a la vez que se realizó en la totalidad de pacientes postmenopáusicas, cirugía no conservadora.

Finalmente se pudieron ver en el estudio otras fortalezas de la cirugía laparoscópica como fueron la ausencia de complicaciones, el bajo promedio de estáncia hospitalaria $(1,8$ días $\pm 0,96)$ y el promedio de costos de hospitalización por paciente $(\$ 482,109.28 \pm$ $\$ 303,459.22$ ).

Analizando los datos de la literatura y los resultados de este trabajo, se concluye, que la laparoscopia es una vía segura, con una baja tasa de complicaciones, (en manos de personal debidamente entrenado) para abordar las masas anexiales sugestivas de benignidad y que lo más importante en el manejo de estas, es tanto la selección objetiva de la paciente, como su estudio y manejo adecuado en todos los casos.

\section{BIBLIOGRAFÍA}

1. Jaroslav F., Parker W., Surrey M., et al. Management of ovarian masses. Journal of Reproductive Medicine July 1992; 37: 559-606.

2. Howard F. Surgical Management of Benign Cystic Teratoma: Laparoscopy Versus Laparotomy. Journal of Reproductive Medicine 1995; 40: 495-99..

3. Albini S., Benadiva C., Haverly K., et al. Management of Benign Ovarian Cystic Teratomas: Laparoscopy Compared With Laparotomy. The Journal of the American Association of Gynecologic Laparoscopists.

4. Mais V., Ajossa S., Piras B., et al. Treatment of Nonendometriotric Benign Adnexal Cysts: A Randomized Comparison of Laparoscopy and Laparotomy. Obstetrics and Gynecology 1995; 86: 770-73.

5. Parker W., Berek J. Management of selected cystic adnexal masses in postmenopausal women by operative laparoscopy: A pilot study. Am J Obstet Gynecol. 1990; 163: 1574-77.

6. Canis M., Mage G., Pouly J., et al. Laparoscopic Diagnosis of Adnexal Cistyc Masses: A 12- Year Experience With Long-Term Follow-Up. Obstetrics and Gynecology. May 1994; 83: 707-12.

7. Herendael B., Beretta P., Slangen T., et al. Management of Adnexal Masses by Operative Laparoscopy. The Journal of the American Association of Gynecologic Laparoscopists. May 1995; 2: 273-77.

8. Parker W., Berek J. Tratamiento de tumores anexiales mediante laparoscopia quirúrgica. Clínicas de Obstetricia y Ginecología de Norteamérica. 1995; 2: 395-403.

9. Sainz de la Cuesta R., Goff B., Fuller A., et al. Prognostic Importance of Intraoperative Rupture of Malignant Ovarian Epithelial Neoplasms.Obstetrics and Gynecology.July1994;84:1-6.

10. Gershenson D., Tortolero-Luna G., Malpica A., et al. Neoplasia Intraepitelial y Cáncer Ováricos. Ginecología y Obstetricia. Temas Actuales. 1996; 2: 435-84.

11. Schwartz P. Utilidad de los marcadores tumorales en el diagnóstico preoperatorio de quistes ováricos. Clínicas de Obstetricia y Ginecología de Norteamérica. 1993; 2: 295-309.

12. Mann W., Reich H. Laparoscopic Adnexectomy in Postmenopausal Women. The Journal of Reproductive Medicine. March 1992; 37: 254-56.

13. Sassone AM., Timor-Tritsch I., Artner A., et al. Transvaginal Sonographic Characterizacion of Ovarian Disease: Evaluation of a New Scoring System to Predict Ovarian Malignancy. Obstetrics and Gynecology. July 1991; 78: 70-6.

14. Prömpeler HJ., Madjar H., Sauerbrei W., et al. Diagnostic Formula for the Differentiation of Adnexal Tumors by Transvaginal Sonography. Obstetrics and Gynecology. March 1997; 89: 428-33.

15. Banacerraf B., Finkler N., Wojciechowski C., et al. The Journal of Reproductive Medicine. May 1990; 35: 491-95.

16. Olt G., Berchuck A., Bast R. The Role of Tumor Markers in Gynecologic Oncology. Obstetrical and Gynecological Survey. 1990; 45: 570-76.

17. Seltzer V. Cirugía laparoscópica de lesiones ováricas: errores potenciales. Clínicas de Obstetricia y Ginecología de Norteamérica. 1993; 2: 385-403.

18. Johns A. Ovariectomía / Ovariocistectomía Laparoscópica. Clínicas de Obstetricia y Ginecología de Norteamérica. 1991; 2: 449-54.

19. Martin D. Tratamiento laparoscópico de endometriomas ováricos. Clínicas de Obstetricia y Ginecología de Norteamérica. 1991; 2: 441-48. 
20. Jaroslav F., Hulka MD., and Carol A. Hulka, MD. Preoperative Sonographic Evaluation and Laparoscopic Management of Persistent Adnexal Masses: A 1994 Review. The journal of the American Association of Gynecologic Laparoscopists. 1994;1:3: 197-203. 